\title{
Determinación Computacional de la Afinidad y Eficiencia de Enlace de Antinflamatorios No Esteroideos Inhibidores de la Ciclooxigenasa-2
}

\author{
Lorena Meneses $^{1}$ y Sebastián Cuesta ${ }^{1}$ \\ 1Laboratorio de Química Computacional, Facultad de Ciencias Exactas y Naturales, \\ Pontificia Universidad Católica del Ecuador, Quito, Ecuador. lmmeneses@puce.edu.ec
}

Recibido: 2015-06-16; aceptado: 2015-08-07

\begin{abstract}
RESUMEN.- El presente es un estudio computacional de la interacción de diferentes antinflamatorios no esteroideos con la enzima Ciclooxigenasa 2 (COX-2). El objetivo fue determinar la afinidad con la cual los inhibidores estudiados se enlazan con el sitio activo de la enzima y calcular la eficiencia de enlace de los mismos. Además, comprobar la aplicabilidad de los métodos de acoplamiento molecular, en la determinación de moléculas prometedoras, que aceleren los estudios de descubrimiento de nuevos fármacos. Se utilizaron métodos de dinámica molecular, para modelar las interacciones entre la enzima COX-2 y los sustratos celecoxib, diclofenaco, etoricoxib, indometacina, ibuprofeno, meloxicam y naproxeno, por medio del programa Autodock VINA. Los resultados muestran que la molécula que posee una mayor afinidad con la COX-2 es el celecoxib, con una energía de enlace de $-10.8 \mathrm{kcal} / \mathrm{mol}$ y una constante de equilibrio Ki de $1.21 \times 10^{-8} \mathrm{M}$. El ibuprofeno y el naproxeno son las moléculas con mayor eficiencia de enlace, con un valor mayor a $-0.48 \mathrm{kcal} / \mathrm{mol} /$ átomos (no hidrógeno). Esto demuestra que una molécula que tiene una buena afinidad, no necesariamente debe tener una buena eficiencia de enlace. Estos valores dan una pauta para poder elegir la mejor molécula para inhibir la enzima COX-2 y en casos de descubrimiento de fármacos, la molécula idónea para invertir en su mejoramiento y optimización, para convertirla en un fármaco aprobado.
\end{abstract}

PALABRAS CLAVES: Afinidad, antinflamatorios no esteroideos, Autodock VINA, COX-2, eficiencia de enlace.

ABSTRACT.- A computational study of the interaction of nonsteroidal antiinflammatory drugs with Cyclooxygenase 2 (COX-2) enzyme is shown. The objective was to determine the affinity of the inhibitors studied with the active site of the enzyme and calculate their binding efficiency. Furthermore, it is intended to check the applicability of the methods of molecular docking in determining promising molecules in order to accelerate drug discovery research. Molecular dynamics methods, using Autodock VINA, were used to model the interactions between the COX-2 enzyme with celecoxib, diclofenac, etoricoxib, indomethacin, ibuprofen, naproxen, and meloxicam. Results show that the molecule with higher affinity for COX-2 is celecoxib with a binding energy of $-10.8 \mathrm{kcal} / \mathrm{mol}$ and an equilibrium constant $\mathrm{Ki}$ of $1.21 \times 10^{-8} \mathrm{M}$. Ibuprofen and naproxen are the molecules with higher binding efficiency with a values greater than $-0.48 \mathrm{kcal} / \mathrm{mol} /$ atoms (other than hydrogen). This shows that a molecule having a good affinity, not necessarily will have a good binding efficiency. These values give researchers a guideline to choose the best molecule to inhibit COX-2 enzyme and in cases of drug discovery processes, the ideal molecule to invest in their improvement and optimization to make it an approved drug.

KEYWORDS: Affinity, Autodock VINA, binding efficiency, COX-2, NSAIDs.

\section{INTRODUCCIÓN}

En la última década, hemos sido testigos de cambios dramáticos en la forma de entender el dolor. En lugar de considerar el dolor como un síntoma de un traumatismo, una infección, una inflamación, o una cirugía, ahora lo tomamos como una entidad patológica discreta que altera fundamentalmente 
todo el sistema nervioso. Avances en tecnologías de neuroimagen, han permitido localizar zonas del cerebro donde se percibe el dolor y por ende, proponer formas más efectivas de tratarlo (Standford Medicine, 2005).

Entre los medicamentos más comunes en el mundo se encuentran los AINEs (Anti Inflamatorios No Esteroideos). Cada día, más de 30 millones de estadounidenses los usan para tratar dolores de cabeza, esguinces, síntomas de la artritis y cualquier otro tipo de molestia (McMurry, 2004).

A un nivel básico, el dolor no es más que el resultado de una señal eléctrica enviada por los nervios al cerebro. Este proceso va acompañado de una liberación de prostaglandinas que ante una lesión, hacen que el tejido se hinche. Estos mediadores químicos amplifican la señal eléctrica procedente de los nervios, causando el dolor (Griffin, 2015). La función de los AINEs es bloquear las enzimas Prostaglandina H2 sintasas, que son las causantes de producir prostaglandinas en el proceso inflamatorio (Hall et al., 2001).

Los mamíferos tienen dos isoformas de la prostaglandina $\mathrm{H} 2$ sintasa, la Ciclooxigenasa 1 (COX1) y la Ciclooxigenasa 2 (COX-2). Ambas poseen en su estructura más de 600 aminoácidos y, aunque tienen similar secuencia de aminoácidos (60 a $65 \%)$, poseen diferentes funciones (Nelson y Cox, 2008).

La COX-2 es la enzima que produce prostaglandinas adicionales que intervienen y causan los efectos en un proceso inflamatorio. Su función es mediar en los procesos de inflamación. Es parte fundamental en el sistema nervioso central (SNC) y el riñón (Hall et al., 2001). No es constitutiva en todos los tejidos, pero puede ser inducida en el sitio de inflamación, manteniendo los mecanismos inflamatorios y amplificando las señales dolorosas (Hall et al., 2001).

En el mercado, existe una gran variedad de medicamentos AINEs. La elección depende entre otros aspectos, del nivel del dolor y la farmacocinética de cada uno (Brunton y Parker, 2008). Dentro del estudio de nuevos medicamentos, se busca que sean eficaces a bajas concentraciones, con alta selectividad y alivio al dolor (Davies y Skjodt, 2000).

El objetivo de un programa de descubrimiento de medicamentos es, generalmente, encontrar una molécula que se una a la proteína deseada, a bajas concentraciones. Cuando un medicamento tiene el valor de la constante de disociación (Ki) muy pequeño, indica que éste y su objetivo biológico se unen fuertemente. Valores ideales de Ki están en el rango de nanomolar (nM). Los valores de Ki y la concentración inhibitoria media (IC50), están relacionados con la potencia de un inhibidor (Stevens, 2014). El IC50 representa la concentración de un medicamento requerida para una inhibición del objetivo biológico del $50 \%$ en pruebas in vitro. Además, la afinidad está relacionada con la energía de enlace, que puede ser determinada a partir de la constante de equilibrio. El valor de esta constante permite además, calcular la energía libre de Gibbs para la unión del complejo medicamento-sitio activo (ecuación 1) (Stevens, 2012).

$$
\begin{aligned}
\mathrm{E}-\mathrm{I} & \frac{K i}{\leftrightarrow} \mathrm{E}+\mathrm{I} \\
\mathrm{Ki}= & \frac{[\mathrm{E}] *[\mathbf{I}]}{[\mathbf{E}-\mathbf{I}]}
\end{aligned}
$$

En los últimos años, se ha vuelto común expresar la energías de unión en términos de eficiencia de enlace (LE). La eficiencia de enlace y propiedades termodinámicas constitutivas del $\Delta \mathrm{G}(\Delta \mathrm{H}$ y $\Delta \mathrm{S})$, pueden proporcionar información sobre la unión de una molécula con su ligando, que van más allá de simples comparaciones de potencia inhibitoria (Reynolds y Holloway, 2011).

La energía libre estándar $\left(\Delta \mathrm{G}^{\circ}\right)$ de unión del complejo enzima-sustrato, puede ser calculada utilizando la ecuación 2. La temperatura usada normalmente es $298 \mathrm{~K}\left(25^{\circ} \mathrm{C}\right)$. Valores más negativos indican mayores energías de unión (Stevens, 2014).

$$
\Delta \mathrm{G}^{\circ}=-2.3 \mathrm{RT} \log (1 / \mathrm{K} i)=2.3 \mathrm{RT} \log (\mathrm{Ki})
$$

Los cambios de entalpía $(\Delta \mathrm{H})$ y de entropía $(\Delta \mathrm{S})$, deben ser considerados en la evaluación de la energía de unión (ecuación 3). Interacciones que son controladas por la entalpía, son los puentes de hidrógeno formados entre el medicamento y la enzima, mientras que efectos hidrofóbicos controlan los cambios en entropía (Stevens, 2014). Cuando un compuesto no polar se encuentra en solución acuosa, las moléculas de agua forman una capa altamente ordenada alrededor de las porciones no polares del compuesto (efecto hidrofóbico) (Stevens, 2012). Una vez que el compuesto entra y se une al sitio activo, algunas moléculas de agua de solvatación pasarán nuevamente a la solución. Las moléculas de agua que están libres en solución tienen más libertad de movimiento que las que están solvatadas. Por lo tanto, la unión de una molécula no polar en un bolsillo lipofílico aumenta el desorden del sistema global (Stevens, 2012). 


$$
\Delta G=\Delta H-\mathrm{T} \Delta S
$$

En el diseño de fármacos, el valor de $\Delta G$ permite evaluar si la modificación de un medicamento aumenta o disminuye la afinidad con su objetivo biológico, estimando la estabilidad relativa de los diferentes compuestos. En síntesis, se puede utilizar para determinar si el sistema está en equilibrio, o qué tan rápido y en qué medida es probable que se dé la reacción de asociación (Stevens, 2012). La importancia descriptiva de $\Delta G$, hace que sea interesante determinar su valor mediante métodos computacionales.

Otro enfoque es analizar el valor de la eficiencia de enlace (LE). LE es una medida que relaciona la energía libre de unión con el tamaño de la molécula. Es importante examinar cómo cambian los valores de LE a medida que se realizan cambios en diferentes partes de la molécula (Murray et al., 2014). La LE se obtiene de dividir la energía libre de unión de cada molécula por el número de átomos $(n)$ no hidrógenos presentes en la estructura (ecuación 4).

$$
\mathrm{LE}=\frac{\Delta \mathbf{G}^{\circ}}{n}
$$

A medida que aumenta la eficiencia de enlace, disminuye el peso molecular del medicamento final (Graham, 2001). La eficiencia de enlace es uno de los índices más usados en la actualidad y se expresa en $\mathrm{kcal} / \mathrm{mol} /$ átomos que no son hidrógeno (Stevens, 2014). Proporciona una idea de la cantidad de energía de enlace por átomo distinto al de hidrógeno. Hay que tener en cuenta que el valor para la eficiencia de enlace debe ser negativo, ya que la energía libre de unión es negativa. Fundamentalmente, LE ayuda a controlar el tamaño molecular de una serie durante la optimización, y da una pauta para decidir entre dos series, cuál es la más prometedora (Murray et al., 2014).

En esta investigación se trabajó con el "software" Autodock VINA. Autodock está diseñado como una herramienta de acoplamiento molecular en la que, mediante modelos matemáticos, se predice la estructura o estructuras de los complejos intermoleculares formados entre dos o más estructuras. Docking, como también se lo llama, es ampliamente usado para sugerir enlaces e inhibir proteínas (Atkinson y Abernethy, 2007). Esta herramienta permite predecir mediante cálculos teóricos, cómo moléculas pequeñas, cómo sustratos y candidatos de fármacos, pueden actuar como ligandos, y se unen a estructuras tridimensionales conocidas, biológicamente importantes (The Scripps Research Institute, 2013).
Al realizar el cálculo del acoplamiento, el software muestra diez resultados diferentes. Cada resultado representa al ligando en una posición espacial distinta. En cada posición se obtiene la afinidad entre la macromolécula y el ligando. El programa muestra las posibles conformaciones del ligando ordenadas según su estabilidad. Para este trabajo, se tomó para cada molécula la conformación de menor energía, que se corresponde con mayor afinidad y por ende, la que tiene mayor probabilidad que suceda en la realidad.

\section{MATERIALES Y MÉTODOS}

Se realizó el modelamiento computacional de la interacción de la enzima COX-2 con moléculas pertenecientes a la misma familia de AINEs (ibuprofeno, naproxeno), diferentes familias (indometacina, diclofenaco, meloxicam) e inhibidores selectivos de la COX-2 (celecoxib, etoricoxib). Se utilizó el programa Autodock VINA. Los AINEs ejercen su acción antinflamatoria, uniéndose al sitio activo ciclooxigenasa, ubicado en la parte inferior de la enzima COX-2. En la Figura 1 se muestra la estructura tridimensional de la COX-2 unida al celecoxib en su sitio activo. La estructura tridimensional (estructura ternaria) de esta enzima, fue obtenida experimentalmente mediante la técnica de rayos $\mathrm{X}$ por otros autores, y sus parámetros estructurales colocados en un
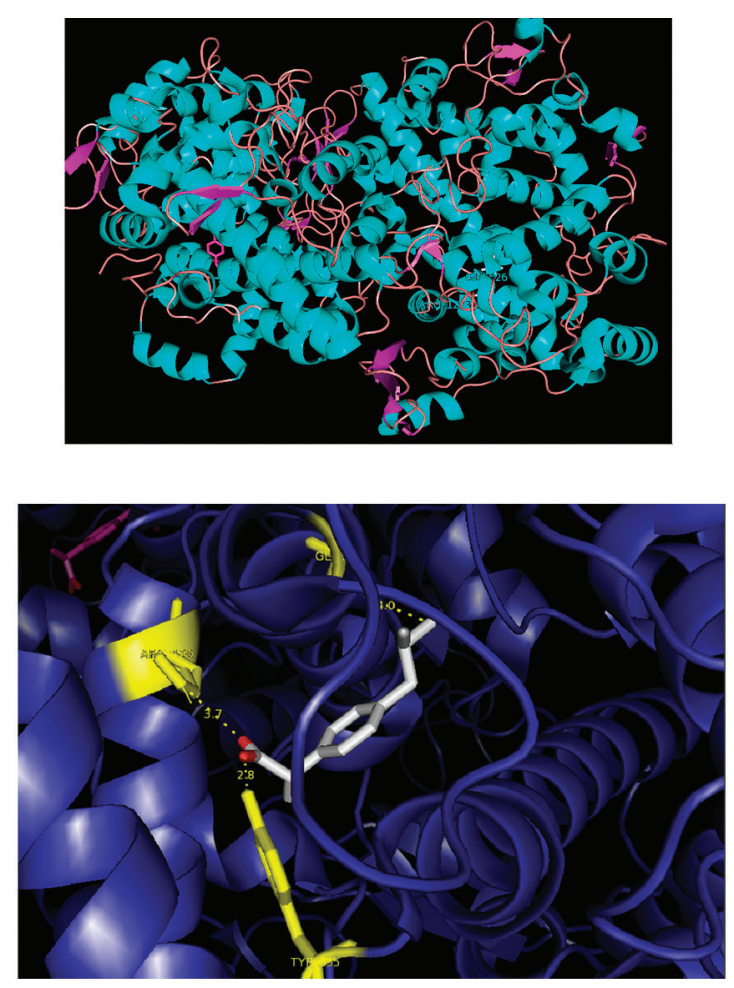

Figura 1. Estructura experimental de la unión de celecoxib con la enzima Ciclooxigenasa-2. 
banco de proteínas (Protein Data Banks), de donde se descargó (Berman et al., 2000), (Figura 1). Para obtener la energía de enlace, se hizo interactuar al ligando con el sitio activo de la macromolécula (COX-2). El programa calcula la energía y produce como resultado varias conformaciones posibles. Los criterios usados para determinar si una conformación es aceptada, o para comparar dos conformaciones, son la energía libre y la distancia media cuadrática (RMSD por sus siglas en inglés) (Kitchen et al., 2004). Dos conformaciones son consideradas idénticas, cuando la diferencia de energía entre ellas está por debajo de las 3kcal/ mol y su RMSD es menor o igual al RMSD promedio menos la desviación estándar. Para conformaciones iguales, las de energías más altas son eliminadas (Ermondi et al., 2004). Con este criterio, se escogió la conformación con la menor energía libre para cada molécula en estudio.

El programa Autodock permite seleccionar, para el cálculo de acoplamiento molecular, cualquier zona de la enzima. En este estudio, se seleccionó el sitio activo ciclooxigenasa de la enzima. El procedimiento que se siguió para cada principio activo fue el siguiente:

- Se obtuvieron las coordenadas de posición de la enzima, del banco de datos de proteínas en formato PDB.

- Mediante el programa Chimera 1.7, se identificaron todos los residuos que no pertenecían a la proteína para poder eliminarlos y que no afecten en los cálculos de docking (Pettersen et al., 2004).

- En Autodock, se abrió Autodock Tools, se escogió la enzima como macromolécula y se la preparó para los estudios, en donde los ligandos, moléculas de solvente y otros residuos fueron removidos del sitio activo de la enzima (Abdel-Azeem et al., 2009). Los átomos de hidrógeno fueron agregados a la estructura con la opción Hydrogen Polar only. Posteriormente, se escogió cada principio activo como ligando y se estableció el área de acoplamiento.

- Finalmente, con los datos obtenidos en Autodock Tools, se creó un archivo de entrada para Autodock VINA (Atkinson y Abernethy, 2007) y se realizó el cálculo. Autodock usa tres métodos para la determinación del sitio activo: un algoritmo genético, un método de búsqueda local y un método global-local adaptado basado en el algoritmo genético de Lamarck en conjunto con campos de fuerza empíricos que permiten obtener las energías libres de unión. (Pouplana et al., 2002).
- Los resultados se visualizaron utilizando el programa Autodock Tools.

\section{RESULTADOS}

En la Figura 2 se muestran las estructuras de todos los ligandos que fueron sometidos al estudio.
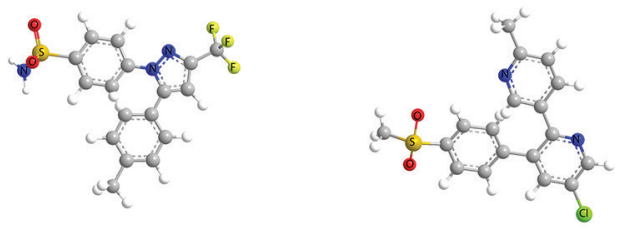

a) Estructura Celecoxib
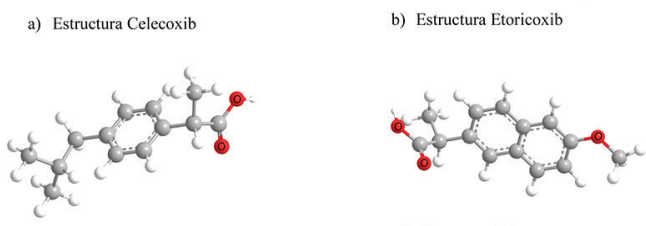

c) Estructura Ibuprofen
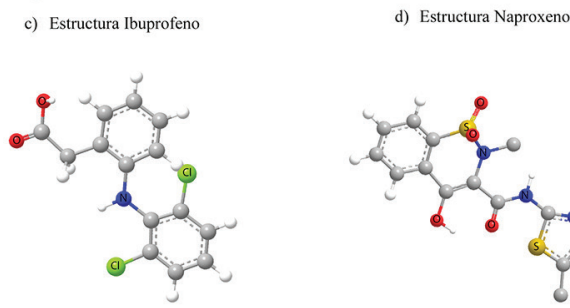

e) Estructura Diclofenaco

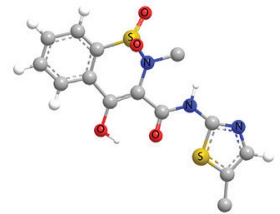

f)Estructura Meloxicam

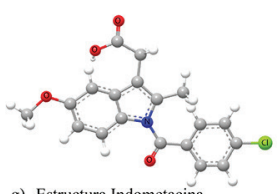

Figura 2. Representación estructural en modelo de bolas de los antinflamatorios no esteroideos estudiados.

Las conformaciones calculadas de cada principio activo, fueron comparadas con un modelo experimental de la interacción entre la COX-2 del ratón común con el medicamento celecoxib, por superposición de las estructuras. El resultado de la comparación se presenta en la Figura 3, donde se pueden observar las conformaciones calculadas de menor energía en color verde para el celecoxib, celeste para el etoricoxib, amarillo para el ibuprofeno, azul para el naproxeno, rojo para el diclofenaco, celeste para el meloxicam y morado para la indometacina. La molécula obtenida de la interacción experimental se muestra de color gris para los átomos de carbono, amarillo para los de azufre, azul para los de nitrógeno y rojo para los de oxígeno.

Con estas conformaciones se obtuvo la energía libre de Gibbs para la unión entre el medicamento y la enzima, que es calculada por el programa y presentada en forma de afinidad. Mediante la 


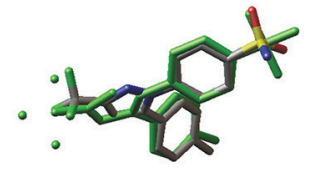

a) Celecoxib

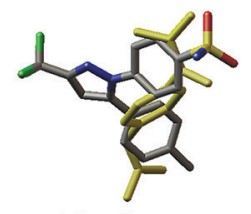

c) Ibuprofeno
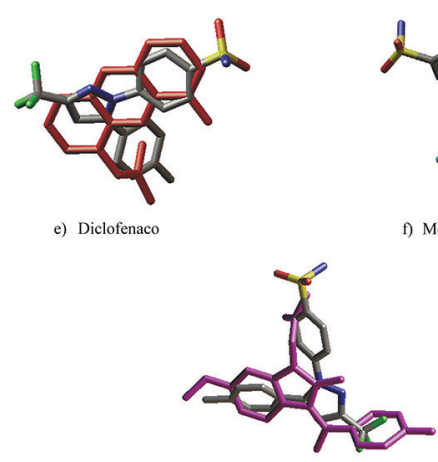

g) Indometacina

Figura 3. Comparación de la conformación de celecoxib obtenida experimentalmente (gris), con las obtenidas computacionalmente para diferentes antinflamatorios no esteroideos.

utilización de la ecuación 2 se obtuvo la constante de equilibrio para cada una de las interacciones. Con la ecuación 4 se obtuvieron los valores teóricos para la eficiencia de enlace. Los valores para estos dos indicadores de afinidad se muestran en la Tabla 1.
Como se puede observar en la Figura 3, todas las conformaciones calculadas de mínima energía, se encuentran superpuestas con la estructura obtenida experimentalmente. En el caso del celecoxib, la molécula se encuentra exactamente en la misma posición que la experimental, lo que nos demuestra la buena correlación de los cálculos computacionales con los experimentales, ya que no solo se encontró el sitio activo, sino también la posición espacial en que la molécula va a entrar e interaccionar con este sitio activo.

En la Tabla 1 se muestra la energía libre de Gibbs para el complejo enzima-sustrato. La afinidad de enlace, representada en la energía libre de Gibbs de un ligando con un receptor, depende de la fuerza de atracción entre los ligandos y sus sitios de unión al receptor. La afinidad de enlace es la fuerza de la interacción entre dos moléculas que se unen de forma reversible (Stevens, 2012). En la Tabla 1 se puede observar que el principio activo que posee mayor afinidad con la enzima COX-2 es el celecoxib, con una energía de enlace de $-10.8 \mathrm{kcal} /$ mol. Luego se encuentra el etoricoxib, seguido del naproxeno, el diclofenaco, el ibuprofeno y al final el meloxicam y la indometacina. El celecoxib tiene también la menor constante de disociación, que se encuentra en el rango nanomolar.

Algunos estudios similares se han realizado utilizando el mismo "software", versiones anteriores de "Autodock" e inclusive diferente "software", obteniendo resultados equivalentes.

Tabla 1. Energía libre de Gibbs y constantes de disociación y eficiencia de enlace para diferentes antiinflamatorios no esteroideos estudiados

\begin{tabular}{ccccc}
\hline Principio activo & Fórmula & $\Delta \mathrm{G}(\mathrm{kcal} / \mathrm{mol})$ & $\mathrm{Ki}(\mathrm{M})$ & $\begin{array}{c}\text { LE kcal/mol/átomos } \\
\text { (no hidrógeno) }\end{array}$ \\
\hline Celecoxib & $\mathrm{C}_{17} \mathrm{H}_{14} \mathrm{~N}_{3} \mathrm{~F}_{3} \mathrm{O}_{2} \mathrm{~S}$ & -10.8 & $1.21^{*} 10^{-8}$ & -0.415 \\
Etoricoxib & $\mathrm{C}_{18} \mathrm{H}_{15} \mathrm{~N}_{2} \mathrm{C}_{1} \mathrm{O}_{2} \mathrm{~S}$ & -9.2 & $1.80^{\star} 10^{-7}$ & -0.383 \\
Ibuprofeno & $\mathrm{C}_{13} \mathrm{H}_{18} \mathrm{O}_{2}$ & -7.7 & $2.26^{*} 10^{-6}$ & -0.513 \\
Naproxeno & $\mathrm{C}_{14} \mathrm{H}_{14} \mathrm{O}_{3}$ & -8.4 & $6.94^{*} 10^{-7}$ & -0.494 \\
Diclofenaco & $\mathrm{C}_{14} \mathrm{H}_{11} \mathrm{NC}_{12} \mathrm{O}_{2}$ & -8.1 & $1.15^{\star} 10^{-6}$ & -0.426 \\
Meloxicam & $\mathrm{C}_{14} \mathrm{H}_{13} \mathrm{~N}_{3} \mathrm{O}_{4} \mathrm{~S}_{2}$ & -6.2 & $2.85^{*} 10^{-5}$ & -0.270 \\
Indometacina & $\mathrm{C}_{19} \mathrm{H}_{16} \mathrm{NC}_{1} \mathrm{O}_{4}$ & -5.3 & $1.30^{*} 10^{-4}$ & -0.212 \\
\hline
\end{tabular}

\section{DISCUSIÓN}

Estudios previos demuestran que las enzimas Ciclooxigenasa poseen dos sitios activos ciclooxigenasa y dos peroxidasa en su estructura (DeWitt, 1999). Los AINEs inhiben los sitios activos ciclooxigenasa que se encuentran en la parte inferior de la enzima. Por este motivo, el modelamiento molecular se enfocó únicamente en la zona del sitio activo ciclooxigenasa.
Al comparar el resultado obtenido con el ibuprofeno $(-7.7 \mathrm{kcal} / \mathrm{mol})$ conel resultado obtenido en otroestudio con la utilización del programa "Autodock 4" (-6.21 $\mathrm{kcal} / \mathrm{mo}$ ) (Savic et al., 2011), se puede observar que la diferencia de energía entre ambas estructuras es de tan solo $1.49 \mathrm{kcal} / \mathrm{mol}$. Tal como se explicó, estructuras se consideran homólogas cuando sus energías difieren en menos de $3 \mathrm{kcal} / \mathrm{mol}$. Aunque no se puede obtener el RMSD, este pequeño cambio en la energía nos da una pauta de la reproducibilidad en los datos. 
En un estudio diferente (Pouplana et al., 2002), utilizando Autodock 3, los autores obtuvieron energías de $-11.04 \mathrm{kcal} / \mathrm{mol}$ para naproxeno, $-11.09 \mathrm{kcal} / \mathrm{mol}$ para diclofenaco y $-10.19 \mathrm{kcal} / \mathrm{mol}$ para indometacina. Aunque los valores difieren en mayor proporción a los obtenidos en este estudio, con excepción de la indometacina, ninguno de los valores sobrepasa la diferencia de $3 \mathrm{kcal} / \mathrm{mol}$. La diferencia en los resultados es comprensible ya que el "software" utilizado es una versión anterior por lo cual algoritmos y cálculos matemáticos pueden ser diferentes. A pesar de esto, se observa buena relación entre resultados con el mismo programa, ya que el naproxeno y el diclofenaco tienen energías similares y la indometacina posee una energía menor en los dos estudios.

Con el programa Merck Molecular Force Field MMFF94x, se obtuvieron energías de $-11.20 \mathrm{kcal} /$ mol para el ibuprofeno y $-12.65 \mathrm{kcal} / \mathrm{mol}$ para el naproxeno (Abdel-Azeem et al., 2009). Las diferencias se dan ya que entre diferentes marcas de "software", se utilizan diferentes algoritmos matemáticos en el cálculo de las conformaciones. Aunque las energías son diferentes, estas no pasan de los $5 \mathrm{kcal} / \mathrm{mol}$. De la misma manera, los resultados son equivalentes entre "softwares" mostrando que el naproxeno tiene una menor energía de enlace que el ibuprofeno en ambos casos.

La afinidad tiene repercusiones en la potencia inhibitoria de la molécula en la enzima (Graham, 2001). Esto es vital en los estudios farmacológicos para determinar la dosis ideal para el medicamento. En las pruebas preliminares para medicamentos, existe un rango muy pequeño donde se alcanza el efecto deseado. Concentraciones elevadas producen toxicidad por sobredosificación, mientras que concentraciones por debajo del rango terapéutico, no llegan a tener ningún efecto en el paciente. Datos de afinidad, sumados a pruebas clínicas, ayudan a determinar la cantidad y el intervalo entre dosificaciones, consiguiendo así, un tratamiento efectivo y seguro.

En la creación de nuevos medicamentos, estos datos son de gran importancia, ya que la mayoría de inhibiciones se dan por competencia entre el sustrato y el medicamento (Murray et al., 2003). En este tipo de competencia, la constante de disociación determina la concentración del medicamento necesaria para lograr una inhibición exitosa de la enzima y el beneficio terapéutico para el paciente. Estos valores ayudan a escoger un compuesto líder, entre un conjunto de potenciales moléculas, para invertir en el desarrollo de nuevos fármacos (Stevens, 2014).
Los datos de afinidad y constante solas, no determinan la potencia global de un medicamento. La potencia es el resultado de la compleja interacción tanto de la afinidad de unión como de la eficiencia de enlace. La eficiencia de enlace es la capacidad del ligando para producir una respuesta biológica a la unión al receptor de destino y la magnitud cuantitativa de esta respuesta (Abad-Zapatero y Metz, 2005). Es una medida de la energía de enlace por átomo de un ligando a su pareja de unión, tal como un receptor o enzima. Al observar estos valores para las moléculas analizadas, podemos ver que el ibuprofeno es la molécula que posee un mayor valor para la eficiencia de enlace, aunque su afinidad no sea la más alta. Esto también sucede con el naproxeno y el diclofenaco. Los inhibidores de COX-2, diclofenaco y naproxeno, aunque tienen LE menores en relación con el ibuprofeno, también son valores que entran dentro de los aceptables, para considerar a una molécula con propiedades farmacológicas interesantes (llamada hit) para estudios posteriores. Con esto se puede normalizar las afinidades de los "hits" para identificar los mejores puntos de partida para la optimización.

Normalizar se refiere a identificar aquellos con los más altos valores de LE, en igualdad de condiciones. A pesar de sus bajas afinidades, fragmentos de los hits seleccionados a menudo tienen LE relativamente buenas $(>0,4)$ a excelentes. Estudios en medicamentos que ya están a la venta (Hopkins et al., 2014), determinaron un LE para los medicamentos orales de $-0.52 \mathrm{kcal} / \mathrm{mol} /$ átomos (no hidrógeno) lo cual está dentro del rango de los resultados obtenidos en este estudio. Además, se muestra como va cambiando la LE a medida que avanza el proceso de descubrimiento siendo para hits $-0,41$, compuestos líderes -0.39 y compuestos en Fase $2-0.42 \mathrm{kcal} / \mathrm{mol} /$ átomos (no hidrógeno). Transformar estos "hits" en compuestos líderes es un desafío que a menudo requiere la adición de 15-20 átomos pesados para aumentar la afinidad. Esto presenta una oportunidad para utilizar diferentes ligandos y la eficiencia de grupos para controlar cuidadosamente las propiedades químicas (Hopkins et al., 2014). La eficiencia de ligando es bastante dependiente del tamaño de la molécula donde ligandos de menor peso molecular van a tener mejores eficiencias en promedio que ligandos más grandes. La principal causa para esto es que a medida que el ligando crece en tamaño se reduce la calidad de la unión entre este y el receptor, ya que ligandos más grandes y complejos complican la entrada hasta el sitio activo del receptor (Reynolds et al., 2008). 
Existen varias limitaciones en el uso de estos descriptores de afinidad. En primer lugar, todos los átomos que no son hidrógeno tienen el mismo peso, por lo que la introducción de un $\mathrm{CH}_{3}, \mathrm{NH}_{2}, \mathrm{OH}, \mathrm{F}, \mathrm{Cl}$, Br creará el mismo cambio en valores de LE, sin tomar en cuenta las ventajas y desventajas de introducir moléculas polares o especies cargadas en la molécula. Esto supone un riesgo en la utilización de LE, sin considerar otras propiedades tales como la potencia, eficiencia de enlace lipofílico (LLE), solubilidad, farmacocinética, entre otros (Murray et al., 2014).

Algunos autores sugieren que existen mejores indicadores de eficiencia ligando, como son el índice porcentual o eficiencia-potencia (PEI, por sus siglas en inglés), el índice de eficiencia de unión (BEI, por sus siglas en inglés), porque son más fáciles de calcular y tienen en cuenta las diferencias entre los elementos en diferentes filas de la tabla periódica (Abad-Zapatero, 2005). La eficiencia de enlace lipofílico (LLE), por otro lado, es un parámetro extremadamente importante que se debe tomar en cuenta durante la optimización de compuestos. LLE considera explícitamente el equilibrio entre la lipofilia y la potencia inhibitoria. Sin embargo, a pesar de sus puntos fuertes, LLE no es tan idóneo cuando se quiere comparar moléculas de diferentes tamaños. También, LLE no es útil cuando el objetivo biológico requiere moléculas muy polares (Murray et al., 2014).

No existe un descriptor de afinidad que sea la solución a todos los problemas y quelleven aléxito. Sin embargo, el concepto de LE es importante en investigaciones, para guiar las estrategias basadas en los fragmentos, para acelerar el descubrimiento de fármacos y en la toma de decisiones (Murray et al., 2014). También se ha utilizado para diseccionar el fragmento más eficiente en moléculas grandes obtenidas de productos naturales (Abad-Zapatero et al., 2010).

La diversidad de mecanismos de enlace de la COX-2 demostrados para diferentes compuestos es impresionante. La habilidad de la enzima de acomodarse estructuralmente a diferentes inhibidores es notable, ya que no existe evidencia de cambios considerables en la conformación de las proteínas en la interacción con AINEs (Blobaum y Marnet, 2007). Los AINEs estudiados, como mostraron los resultados, bloquean la unión entre el ácido araquidónico con el sitio activo ciclooxigenasa de la enzima (impidiendo la formación de la prostaglandina). Esta inhibición se da por competencia, por lo que esta puede ser revertida al diluir el inhibidor, o aumentando la cantidad de sustrato (Nelson y Cox, 2008). La cantidad de antinflamatorio necesaria para lograr la inhibición depende de los valores de afinidad obtenidos en el cálculo, así como de otras propiedades farmacocinéticas como biodisponibilidad, unión proteica, vida media y de la interacción del fármaco con otros sistemas biológicos del cuerpo. Tomando en cuenta los datos obtenidos, se puede determinar que los inhibidores selectivos de la COX-2, debido a sus energías más bajas, van a ser más útiles para tratar dolores más agudos, en comparación al resto de AINEs estudiados. Así, mientras se necesitan 120 mg ( $3 \times 10^{-4}$ moles) de etoricoxib al día, para tratar dolores como artritis gotosa aguda, tratamientos con ibuprofeno pueden llegar a los $800 \mathrm{mg}\left(4 \times 10^{-3}\right.$ moles) al día (American Society of Health-System Pharmacists, 2015).

Al comparar el ibuprofeno con el meloxicam, podemos observar que aunque el ibuprofeno posee valores más favorables de energía libre de Gibbs y de eficiencia de enlace, la dosis diaria de meloxicam es de tan solo $15 \mathrm{mg}$, mientras la del ibuprofeno es de 400 a $600 \mathrm{mg}$. Esto se debe a la influencia que tienen otras propiedades farmacocinéticas, en este caso la vida media del medicamento, que mientras en el ibuprofeno es de tan solo 1.8 horas, en el meloxicam puede llegar a las 20 horas, siendo necesario menor cantidad del medicamento para lograr mantener la dosis terapéutica en el cuerpo (American Society of Health-System Pharmacists, 2015).

Las comparaciones analizadas son útiles para determinar la aplicabilidad y exactitud de los resultados obtenidos mediante programas computacionales, en la búsqueda de sitios activos, compuestos con propiedades farmacológicas interesantes y enlaces enzima-sustrato de objetivos biológicos y enzimas de interés.

\section{CONCLUSIONES}

-El principio activo que posee mayor afinidad con la enzima COX-2 es el celecoxib, con una energía de enlace de $-10.8 \mathrm{kcal} / \mathrm{mol}$ y una constante de equilibrio $K i$ de $1.21 \times 10^{-8} \mathrm{M}$.

- El ibuprofeno y el naproxeno son las moléculas con mayor valor de eficiencia de enlace que sobrepasa los $-0.48 \mathrm{kcal} / \mathrm{mol} /$ átomos (no hidrógeno).

- Aunque los datos de $\Delta G, K i$ y LE son fundamentales para iniciar un estudio en una molécula determinada, otras propiedades como biodisponibilidad, tiempo de vida media y unión proteica, son importantes en el momento de definir la dosis del medicamento para el paciente, por lo cual este estudio debería complementarse con pruebas clínicas en el laboratorio, para poder determinar seguridad y dosis terapéutica. 


\section{AGRADECIMIENTOS}

A la DGA-PUCE, a través del proyecto "Estudio téorico y experimental en síntesis orgánica (Ibuprofeno)", con código J13075.

\section{REFERENCIAS BIBLIOGRÁFICAS}

Abad-Zapatero C y Metz J. 2005. Ligand efficiency indices as guideposts for drug discovery. Drug Discovery Today, 10 (7): 464-469.

Abad-Zapatero C, Perišić O, Wass J, Bento P, Overington J, Al-Lazikani B y Johnson M. 2010. Ligand efficiency indices for an effective mapping of chemico-biological space: the concept of an atlas-like representation, Drug Discovery Today, 15: 804-811.

Abdel-Azeem A, Abdel-Hafez A, El-Karamany G y Farag H. 2009. Chlorzoxazone esters of some non-steroidal anti-inflammatory (NSAI) carboxylic acids as mutual prodrugs: Design, synthesis, pharmacological investigations and docking studies. Bioorganic \& Medicinal Chemistry, 17: 3665-3670.

American Society of Health-System Pharmacists. 2015. Consumer Medication Information. Página de Internet: http://www.nlm.nih. gov / medlineplus / spanish / druginfo/ meds / a689002-es.html Consultada 06-junio-2015.

Atkinson A y Abernethy D. 2007. Principles of Clinical Pharmacology. Segunda edición. Elsevier Inc. UK. 146-149 pp.

Berman H, Westbrook J, Feng Z, Gilliland G, Bath T, Weissig H, Shindyalov I and Bournel P. 2000. The Protein Data Bank, Oxford Journals Nucleic Acids Research, 28 (1): 235-242.

Blobaum A y Marnett L. 2007. Structural and Functional Basis of Cyclooxygenase Inhibition, Journal of Medicinal Chemistry, 50 (7): 1425-1441.

Brunton L y Parker K. 2008. Goodman \& Gilman's Manual of Pharmacology and Therapeutics. Décimo primera edición. McGraw-Hill. USA. 421, 428, 429, 436, 451 pp.

Davies NM y Skjodt NM. 2000. Choosing the right nonsteroidal anti-inflammatory drug for the right patient: a pharmacokinetic approach, Clinical Pharmacokinetics, 38 (5): 377-392.

DeWitt D. 1999. Cox-2-Selective Inhibitors: The New Super Aspirins, Molecular Pharmacology, 55: 625-631.
Ermondi G, Caron G, Lawrence R y Longo D. 2004. Docking studies on NSAID/COX-2 isozyme complexes using Contact Statistics analysis. Journal of Computer-Aided Molecular Design, 18: 683-696.

Graham P. 2001. An Introduction to Medicinal Chemistry. Quinta edición. Oxford. Reino Unido. 116, 211 pp.

Griffin M. 2015. Pain Relief: How NSAIDs Work. Página de Internet: http: / / www.webmd.com/ arthritis / features / pain-relief-how-nsaidswork Consultada 03-Junio-2015.

Hall V, Murillo N, Rocha M y Rodríguez E. 2001. Antinflamatorios No Esteroidales. Centro Nacional de Información de Medicamentos, Instituto de Investigaciones Farmacéuticas. Universidad de Costa Rica, Costa Rica. 7: 13,14 pp.

Hopkins A, Keserú G, Leeson P, Rees P y Reynolds C. 2014. The Role of Ligand Efficiency Measures in Drug Discovery, Nature reviews drug discovery, 13 (2): 105-121.

Kitchen D, Decornez H, Furr J y Bajorath J. 2004. Docking and scoring in virtual screening for drug discovery: methods and applications. Nature reviews: Drug discovery, 3: 935-949.

McMurry, J. 2004. Organic Chemistry, sexta edición. Thomson, USA. 305, 519, 1034, 1035 pp.

Murray C, Erlanson D, Hopkins A, Keseru G, Leeson P, Rees D, Reynolds C y Richmond N. 2014. Validity of Ligand Efficiency Metrics, ACS Medicinal Chemistry Letters, 5: 616-618.

Murray R, Granner D, Mayes P y Rodwell V. 2003. Harper's Illustrated Biochemistry. Vigésima sexta edición. McGraw-Hill. USA. 49, 50, 190$194 \mathrm{pp}$.

Nelson D y Cox M. 2008. Lehninger Principles of Biochemistry. Quinta edición. W.H Freeman and Company. USA. 184, 358, 817, 1184 pp.

Pettersen E, Goddard T, Huang C, Couch G, Greenblatt D, Meng E y Ferrin T. 2004. UCSF Quimera - a visualization system for exploratory research and analysis. Journal of Computational Chemistry, 25 (13): 1605-1612.

Pouplana R, Lozano J y Ruiz J. 2002. Molecular modelling of the differential interaction between several non-steroidal anti-inflammatory drugs 
and human prostaglandin endoperoxide $\mathrm{H}$ synthase-2 (h-PGHS-2). Journal of Molecular Graphics and Modelling, 20: 329-343.

Reynolds C, Tounge B y Bembenek S. 2008. Ligand Binding Efficiency: Trends, Physical Basis, and Implications J. Med. Chem, 51: 2432-2438.

Reynolds C y Holloway M. 2011. Thermodynamics of Ligand Binding and Efficiency, ACS Medicinal Chemistry Letters, 2: 433-437.

Savic J, Dilber S, Markovic B, Milenkovic M, Vladimirov S y Juranic I. 2011. Docking Studies and a-Substitution Effects on the Anti-Inflammatory Activity of $B$-Hydroxy-ßarylpropanoic Acids. Molecules, 16: 6645-6655.

Standford Medicine. 2005. Pain Research at Stanford. Página de Internet: http://med. stanford.edu/paincenter/research.html Consultada 03-junio-2015.
Stevens E. 2012. Medicinal Chemistry: The Modern Drug Discovery Process. Primera edición. Pearson. USA. 106, 254 pp.

Stevens E. 2014. 001 x: Medicinal Chemistry: The molecular basis of drug discovery, a course of study offered by Davidson College through edX platform, disponible en www.edx.org, chapters 4,$1 ; 4,3 ; 9,1 ; 10,2$. Consultada 11-febrero-2015.

The Scripps Research Institute. 2013. Autodock. Página de Internet: http:/ /autodock.scripps. edu /, 24/09/2013 Consultada 03-junio-2015. 